\title{
Glycan analysis of colorectal cancer samples reveals stage-dependent changes in CEA glycosylation patterns
}

Qianqian Zhao ${ }^{1,2 \dagger}$, Tiancheng Zhan ${ }^{3 \dagger}$, Zaian Deng ${ }^{1}$, Qianqian $\mathrm{Li}^{1}$, Yaming Liu' ${ }^{1}$, Shaojie Yang ${ }^{4}$, Dengbo $\mathrm{Ji}^{3}$ and $\operatorname{Yan} \mathrm{Li}^{1,2^{*}}$

\begin{abstract}
Background: Carcinoembryonic antigen (CEA) is a glycoprotein associated with colorectal cancer (CRC). While the functions of its gene and protein have been fully characterized, its post-translational modifications in the context of CRC development remain undefined.

Methods: To show the correlation between the different stages of CRC development and changes in the glycosylation patterns of CEA, we analyzed CEA in tumor tissues (CEA-T) and paired tumor-adjacent normal tissues (CEA-A) from 53 colorectal cancer patients using a high-density lectin microarray containing 56 plant lectins.

Results: We detected higher expression levels of fucose, mannose and Thomsen-Friedenreich antigen, and lower expression levels of $\mathrm{N}$-acetylgalactosamine, $\mathrm{N}$-acetylglucosamine, galactose, branched and bisecting $\mathrm{N}$-glycans on CEA in the tumor tissues relative to the tumor-adjacent normal tissues. Furthermore, a combinatorial assessment of 9 lectins is sufficient to distinguish CRC tumor tissues from tumor-adjacent normal tissues with 83\% sensitivity and $\sim 90 \%$ specificity. Moreover, the levels of $\mathrm{N}$-acetylgalactosamine, mannose, galactose, $\mathrm{N}$-acetylglucosamine on CEA showed a downward trend after first experiencing an increase at Stage II with the stages of CRC.
\end{abstract}

Conclusions: Our insights into the changing CEA glycosylation patterns and their role in the development of CRC highlight the importance of glycan variants on CEA for early clinical detection and staging of CRC.

Keywords: Colorectal cancer, Carcinoembryonic antigen, Glycosylation, Lectin microarray

\section{Background}

Colorectal cancer (CRC) is the third most common diagnosed cancer globally [1]. CRC shows little symptoms in its early stage, resulting in regional or distant metastasis in most patients at the time of diagnosis, rendering treatment difficult [2]. Development of CRC occurs progressively, usually spanning $5-10$ years. This extended timeframe provides ample opportunities for treatment, especially during the early stage (including the high-risk stage II) [3-5]. However, current screening methods are

\footnotetext{
*Correspondence: yanli@ibp.ac.cn

†Qianqian Zhao and Tiancheng Zhan contributed equally to this work ${ }^{1}$ Laboratory of Interdisciplinary Research, Institute of Biophysics, Chinese Academy of Sciences, 15 Datun Road, Chaoyang District, Beijing 100101, China

Full list of author information is available at the end of the article
}

of low sensitivity and specificity [6]. Recently, genomic and proteomic studies found new candidate biomarkers for detecting the early stage of CRC, however, none has so far been tested in clinical trials $[7,8]$. Therefore, a better understanding of the biology of CRC is paramount to more reliably predict, diagnose and monitor the disease, and to ultimately find efficient drug targets.

Glycosylation is one of the major post-translational modifications found in proteins. It alters protein function and plays an important role in many different biological processes, including protein-protein interactions, cellcell recognition, adhesion and migration [9-11]. Aberrant glycosylation is associated with the occurrence and progression of various tumors [12]; it may be a result of initial oncogenic transformation, as well as a key event in induction of invasion and metastasis [13]. Changes in 
glycosylation patterns correlate well with the progression of colorectal cancer through its different stages, and have been found for $\mathrm{O}$-glycans, $\mathrm{N}$-glycans, globo-type glycosphingolipid (GLS)-glycans, sialylation, (Sialyl) Lexis antigens [14]. Importantly, changes in glycan modifications appear to occur more frequently than changes in the actual protein concentration [15, 16]. Thus, analysis of changes in glycosylation patterns associated with a particular protein should yield biomarkers relevant to effective cancer diagnosis. For instance, the core fucosylation of $\alpha$-fetoprotein has recently been approved as a biomarker for the early detection of hepatocellular carcinoma (HCC), distinguishing it from chronic hepatitis and liver cirrhosis [17].

Human carcinoembryonic antigen (CEA) is the most frequently used marker for colorectal cancer screening, diagnosis and monitoring. Due to a lack of sensitivity and specificity, however, its clinical application has remained limited. CEA is a stable glycoprotein consisting of 60\% carbohydrate and a molecular mass of $\sim 180-200 \mathrm{kDa}$. The carbohydrate side chains of CEA are highly variable, most of which being composed of mannose, galactose, $\mathrm{N}$-acetylglucosamine, fucose and sialic acid [18]. Recently, we examined glycans in colorectal carcinoma tissue samples, and identified $61 \mathrm{~N}$-glycoforms present on the surface of CEA. In one of our recent studies, we showed that the composition of the glycans associated with CEA displays a considerable heterogeneity [19]. Saeland et al. [20] compared CEA glycosylation patterns of normal and colorectal cancer tissues and found that Lewis $\mathrm{X}$, Lewis $\mathrm{Y}$, mannose and branched $N$-glycans are increased in tumor-associated CEA. However, a more detailed understanding of the changes in CEA protein surface glycans, especially with the development of CRC is still lacking. Importantly, analysis of carbohydrate expression profiles of CEA with the progression of CRC is crucial for the understanding of the biology of tumor growth, proliferation, and metastasis, and should aid the development of novel cancer biomarkers for early diagnosis of CRC.

Lectin is a protein found in both plants and animals that specifically binds glycan; because of this specific binding property, it has been used for glycan detection as a part of various techniques including affinity chromatography and lectin blots [21]. Lectin microarray technology is a rapid and high-throughput platform for analyzing glycosylation patterns of specific glycoproteins in clinical samples [22]. It allows simultaneous profiling of hundreds of lectins in a single screening of multiple biological samples [23]. Thus, lectin-based glycan detection methods provide a broad picture of the glycan structures present on proteins, and have been used to study changes of glycans in various diseases [24].
Here we set out to perform glycosylation profiling for CEA between tumor tissues (CEA-T) and tumor-adjacent normal tissues (CEA-A) using a high-density lectin microarray. We found that changes in the glycosylation patterns of CEA correlated well with CRC tumorigenesis and progression, with specific glycans being differently expressed on CEA in a stage-dependent manner.

\section{Methods \\ Materials}

Lectin microarrays were purchased from BCBIO (Guangzhou, China). Commercial standard Carcinoembryonic Antigen (CEA) was purchased from LEE BioSolutions, Inc (St. Louis, MO). Human Carcinoembryonic Antigen (CEA) ELISA Kit was purchased from Yu Ping biotechnology (Shanghai, China). Anti-Human CEA antibody was purchased from eBioscience Inc (San Diego, CA). Rabbit anti-mouse IgG-Alexa Fluor 647 conjugate was purchased form Invitrogen (Eugene, OR). The incubation chamber and holder for the lectin microarray were purchased from Whatman Schleicher and Schuell (Keene, $\mathrm{NH})$. Sodium periodate was purchased from Bio-Rad Laboratories (Hercules, CA). 4-(4- $\mathrm{N}$-maleimidophenyl) butyric acid hydrazide hydrochloride (MPBH) was from Thermo Fisher Scientific, Inc. (Rockford, IL). All other chemicals and reagents were purchased from SigmaAldrich (St.Louis, MO).

\section{Specimen}

Samples and clinical information were reviewed and approved by the Institutional Review Board of Institute of Biophysics, Chinese Academy of Sciences. After obtaining signed informed consent, tumor tissues and paired tumor-adjacent normal tissues from 53 patients with colorectal carcinoma (stage I-IV) were collected from Beijing Cancer Hospital (patient information as listed in Table 1). All patients enrolled in the study had operative treatment of colorectal carcinoma and the surgical pathology report was used to confirm the diagnosis of UICC/AJCC' stage of colorectal carcinoma. Each sample was immediately placed on the ice after procurement and stored at $-80^{\circ} \mathrm{C}$. All tissue samples were thawed less than three times prior to extraction in order to minimize variability introduced by that process [22].

\section{Protein extraction and determination of CEA concentration} Colorectal carcinoma tissues were quickly removed from the cryovial and washed using PBS buffers. Tissues were cut into pieces and weighed. $1 \mathrm{ml}$ RIPA lysis buffer ( $0.15 \mathrm{~g}$ Tris, $0.438 \mathrm{~g} \mathrm{NaCl}, 0.05 \mathrm{~g} \mathrm{NaOH}, 0.5 \mathrm{~g}$ Sodiumdeoxycholate and $0.05 \mathrm{~g}$ SDS) was added to the $100 \mathrm{mg}$ tissue pieces. The mixture was grinded into homogenate in a tissue grinder. All tissue homogenates were incubated 
Table 1 Detailed information of participating colorectal cancer patients

\begin{tabular}{|c|c|c|c|c|c|}
\hline Stage & Gender M/F & Age (mean \pm SD) & Other diseases (yes/no) & $\begin{array}{l}\text { Adjacent tissue } \\
\mathrm{C}_{(\text {CEA) }}=0.25 \mu \mathrm{g} / \mathrm{ml}\end{array}$ & $\begin{array}{l}\text { Tumor tissue } \\
C_{\text {(CEA) }}=0.25 \mu \mathrm{g} / \mathrm{ml}\end{array}$ \\
\hline I (17) & $6 / 11$ & $58 \pm 12$ & $10 / 7$ & 17 & 17 \\
\hline ॥ (14) & $7 / 7$ & $63 \pm 12$ & $6 / 8$ & 14 & 14 \\
\hline III (9) & $5 / 4$ & $63 \pm 10$ & $5 / 4$ & 9 & 9 \\
\hline IV (13) & $9 / 4$ & $65 \pm 10$ & $11 / 2$ & 13 & 13 \\
\hline
\end{tabular}

$M$, male; $F$, female; $C_{(C E A)}$, the concentration of $C E A ; S D$, standard deviation

and then centrifuged at $15,000 \mathrm{~g}$ for $15 \mathrm{~min}$, the supernatant was kept at $-80{ }^{\circ} \mathrm{C}$. Subsequently, the concentration of CEA was determined using Human Carcinoembryonic Antigen (CEA) ELISA Kit [19].

\section{Lectin microarray}

The lectin microarray was first blocked in $50 \mathrm{mM}$ ethanolamine in borate buffer $(\mathrm{pH} \mathrm{8.0)}$ for $1 \mathrm{~h}$ at room temperature. The slide was then washed once in TBS with $0.1 \%$ Tween20 (TBST 0.1), followed by two washes in TBS and dried by spinning at $500 \mathrm{~g}$ for $5 \mathrm{~min}$. Standard CEA was diluted into $100 \mu \mathrm{l}$ using TBS buffer (for concentrations of $0,0.1,0.5,1,5,10 \mu \mathrm{g} / \mathrm{ml}$ respectively). The samples were allowed to bind on lectin microarray and incubated at room temperature for $6 \mathrm{~h}$. The primary antibody (mouse anti-human CEA antibody) and the secondary antibody (rabbit anti-mouse IgG-Alexa Fluor 647 conjugate) were mixed with $20 \mathrm{mM}$ sodium periodate at $4{ }^{\circ} \mathrm{C}$ for $1 \mathrm{~h}$ in the dark to oxidize sugar groups. The oxidized glycans of antibodies were then blocked with $1 \mathrm{mM}$ 4-(4- $\mathrm{N}$-maleimidophenyl) butyric acid hydrazide hydrochloride (MPBH) for $2 \mathrm{~h}$ followed by $1 \mathrm{mM}$ CysGly dipeptide in $4{ }^{\circ} \mathrm{C}$ overnight [24]. The microarray was removed from the incubation chamber, and then $2 \mu \mathrm{g} / \mathrm{mL}$ oxidized mouse anti-human CEA antibody was sequentially hybridized with the microarray at $4{ }^{\circ} \mathrm{C}$ overnight. After washing, $2 \mu \mathrm{g} / \mathrm{ml}$ oxidized rabbit anti-mouse IgG-Alexa Fluor 647 conjugate was hybridized for $1 \mathrm{~h}$ with gentle shaking. After washing with TBST buffer, the microarray washed twice with water. The array was dried by spinning at $500 \mathrm{~g}$ for $5 \mathrm{~min}$, and scanned using a LuxScan $^{\text {TM }} 10 \mathrm{~K}-\mathrm{A}$ scanner at $10 \mu \mathrm{m}$ resolution. The scanning condition was set to 85 power and 850 PMT value for Cy5 channel. The scanned images were analyzed using LuxScan 3.0 software to convert to numerical format (GPR) using a homemade "GAL" files [25].

For clinical samples testing, CEA in all samples were diluted with TBS buffer into the same concentration. The same amount of CEA protein of each sample was applied to lectin microarray using the protocol described above. TBS buffer without protein was used as negative control.

\section{Data analysis}

The mean of the foreground spot intensity and mean of the background spot intensity were used in this analysis. The signal-to-noise ratio (the mean of spot foreground intensity relative to the mean of spot background intensity) of each lectin spot was used to calculate each lectin [25]. Because each lectin was present in triplicate, the signal intensities from replicate lectin measurements within the same array were averaged $(\mathrm{CV} \leq 30 \%)$. Each lectin microarray contains a negative control sample, the $95 \%$ confidence interval of the signal-to-noise of all lectins is $(0.8,1.2)$. The signal-to-noise ratio of greater or equal to 1.2 was defined as a positive signal. Any undetected signal was set to 1 . All positive and negative signals of tissue samples were used in all subsequent data analysis.

Significant differences between CEA-A and CEA-T of colorectal carcinoma patients were tested using a twotailed paired $t$ test. The bars represent the mean values with standard error of mean (SEM). One-way analysis of variance (ANOVA) test was used to the differences between four stages. $P$ values lower than 0.05 were considered as statistically significant.

\section{Results}

\section{Sensitivity of lectin microarray}

First, we established a procedure to determine CEA glycosylation patterns using a lectin microarray (Fig. 1a). As shown in Fig. 1b, the microarray contains 56 lectins, with each lectin present in triplicate. All lectins used possess diverse glycan specificities as defined in previous studies (see Additional file 1: Table S1).

Next, in order to optimize conditions for our lectin microarray procedure, we evaluated its sensitivity using commercial standard CEA purified from human liver metastases. We incubated the microarray with a series of CEA concentrations (keeping the volume of each sample at $100 \mu \mathrm{l}$ ). As shown in Additional file 2, we showed that at the highest concentration of CEA $(10 \mu \mathrm{g} / \mathrm{ml}), 31$ lectins specifically interacted with CEA. As shown in Fig. 1c, the signal of each detectable lectin spot increased with the concentration of CEA. In order to determine the optimal 


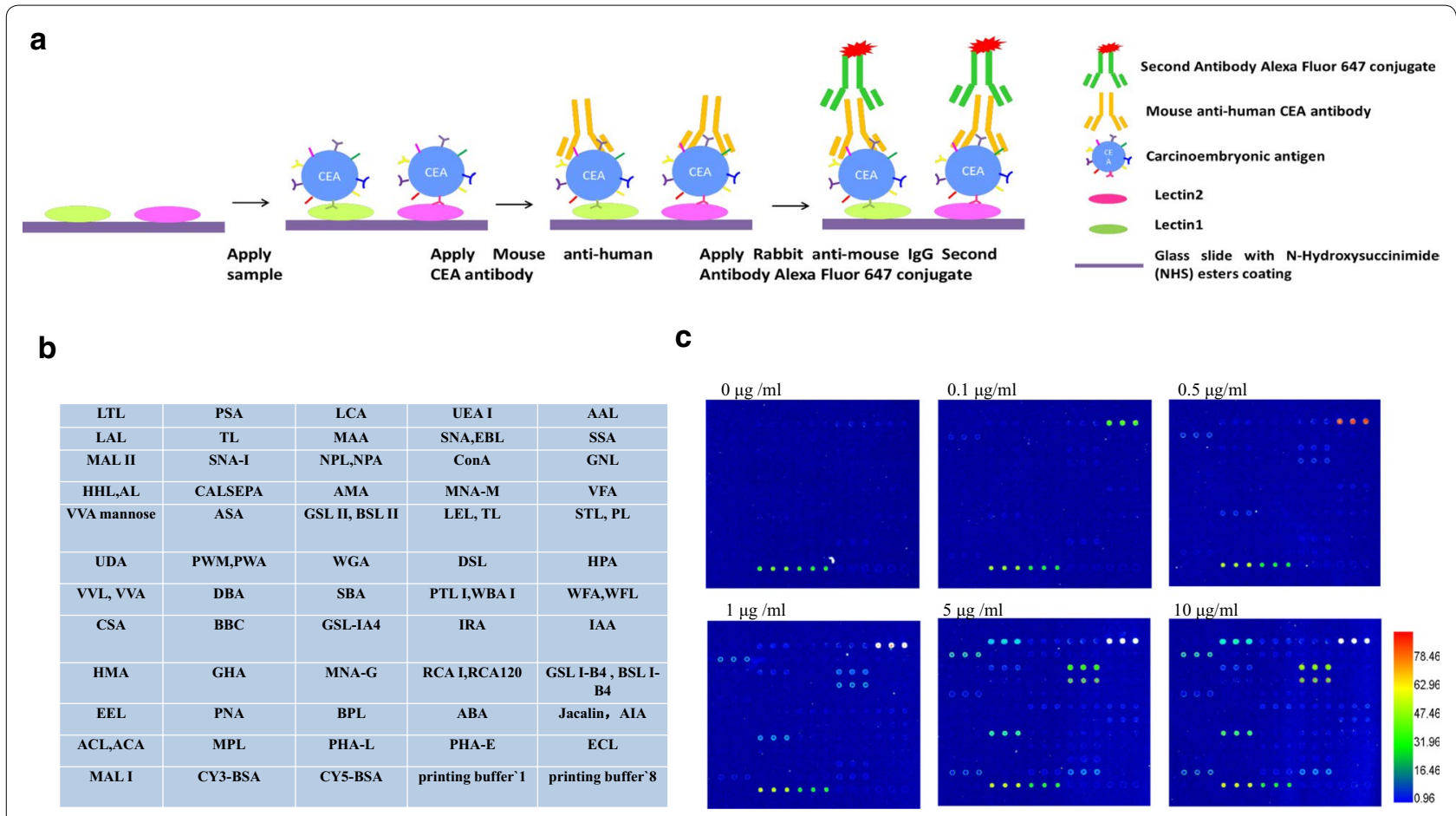

d

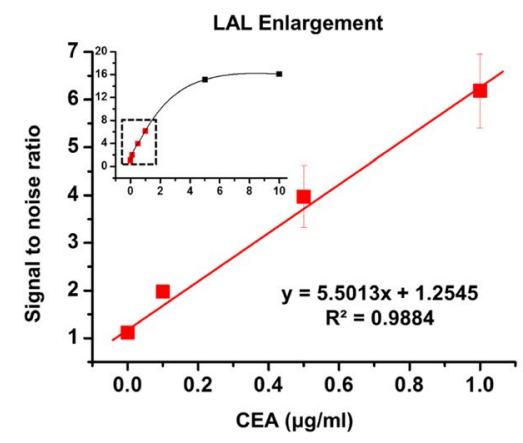

ACL ACA Enlargement

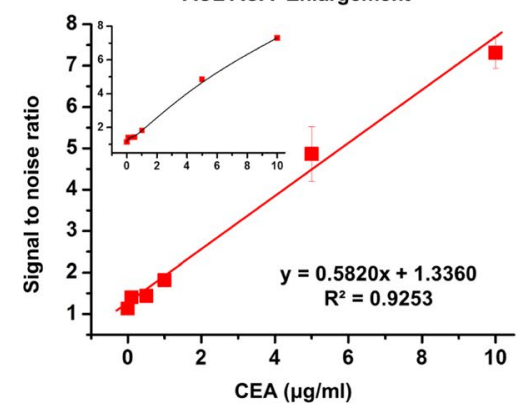

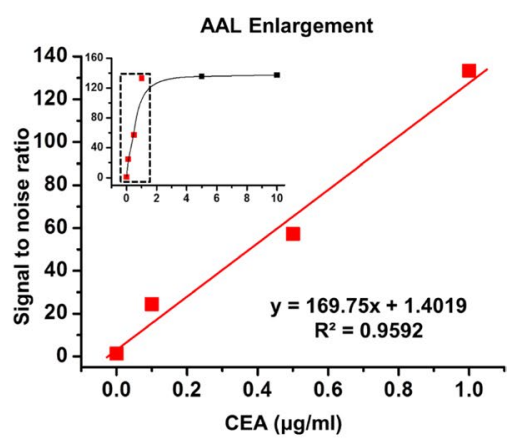

PHA-L Enlargement

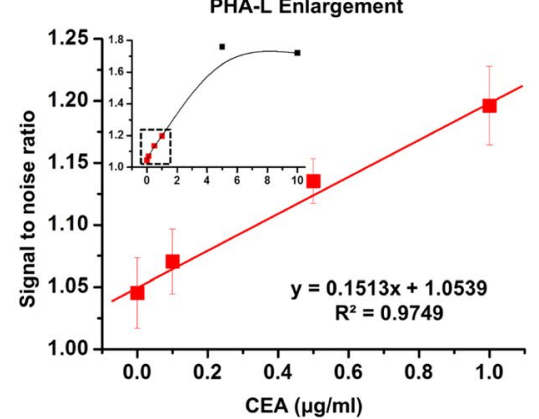

Fig. 1 Identification of lectins interacting with purified CEA. a Schematic presentation of lectin microarray for CEA glycosylation analysis. Lectin 1 and 2 bind two different glycosylation patterns of CEA. b Design of the lectin microarray containing 56 lectins. c Representative lectin microarray binding patterns of six different CEA concentrations. $\mathbf{d}$ Four representative lectins bind different concentrations of CEA. The smaller diagrams show the overall changes with the increase of CEA concentration. The red points in the dashed box show expanded portions of the small diagrams. Bars represent the mean values with standard deviation (SD). CY3-BSA and CY5-BSA are positive controls. Printing buffer 1 and 8 serve as negative controls. The color bar represents corresponding signal-to-noise value 
amount of clinical sample loaded, we analyzed fluorescence signal intensity for the 31 lectins as a function of CEA concentration. Four calibration curves of representative lectins are shown in Fig. 1d. Together, these results indicate that below a CEA concentration of $0.5 \mu \mathrm{g} / \mathrm{ml}$, the signal-to-noise ratio of the assay is positively correlated with the concentration of CEA.

\section{Glycosylation pattern analysis of CEA-A and CEA-T using lectin microarray}

To compare the glycosylation patterns of CEA-A and CEA-T, we collected CRC tumor and paired tumor-adjacent normal tissues from 53 patients at different stages ( $\mathrm{n}=17$, stage $\mathrm{I} ; \mathrm{n}=14$ stage $\mathrm{II} ; \mathrm{n}=9$ stage III; $\mathrm{n}=13$ stage IV). The detail information of these patients was shown in Table 1 . We excluded the possibility that other diseases of patients skewed experimental results (see Additional file 3). The concentrations of CEA in tissue lysates were determined using an ELISA assay; the data was shown in Additional file 4: Table S2. In order to make sure the amount of CEA in each clinical sample was identical, we adjusted CEA concentrations to $0.25 \mu \mathrm{g} / \mathrm{ml}$, and maintained sample volumes at $200 \mu \mathrm{l}$.

As shown in Fig. 2a, we observed 22 lectins binding CEA had significant difference between CEA-T and CEA-A. Lectins AAL, MNA-M, Con A, GNL, AMA, HHL (AL), VVA Man, NPL (NPA), PSA and ACA binding to CEA were higher in CRC tumor tissues relative to tumor-adjacent normal tissues. The results suggest that fucose, mannose and the Thomsen-Friedenreich antigen (TF-antigen) (Core1, Galß1-3 GalNAc-Ser/Thr) are higher expressed on CEA-T than CEA-A. Moreover, lectins HPA, SSA, BBC, IRA, IAA, MPL, RCA-C (RCA 120), HMA, PHA-L, PHA-E, STL (PL) and WGA binding to CEA were lower in CRC tumor tissues relative to tumor-adjacent normal tissues. The results suggest that $\mathrm{N}$-acetylgalactosamine, galactose, $\mathrm{N}$-acetylglucosamine, branched $\mathrm{N}$-glycans and bisecting $\mathrm{N}$-glycans are lower expressed on CEA-T than CEA-A.

To directly compare the binding of 22 lectins (see Fig. 2a for all lectins) to CEA, we generated and clustered a heat map according to lectin-binding pattern and intensity. As shown in Fig. 2b, lectin patterns showed considerable differences between CRC tissues and tumor-adjacent normal tissues. This suggests that the changes of CEA surface glycans correlated well with the presence of CRC. Different lectins with the same glycan binding specificity were clustered into one group. For example, lectins binding to mannose, such as GNL, MNA-M, NPL (NPA), AMA, VVA Man and HHL (AL), were grouped into one cluster.

In order to evaluate the discriminative power of lectin binding to CEA-associated glycosides for the purpose of distinguishing CRC tumor tissues from tumor-adjacent normal tissues, we performed ROC analysis (Fig. 3a). Our analysis showed that the values of area-under-the-curve (AUC) of 9 lectins (HPA, WGA, AAL, PHA-L, BBC, SSA, MNA-M, Con A, PHA-E) binding to CEA were greater or equal to 0.7 , which suggested that these lectins have moderate accuracy for detecting CRC tumor tissues from tumor-adjacent normal tissues. Next, we analyzed the combined panel of these 9 lectins using binary logistic regression rule. The AUC of combined lectins was 0.901 with $83 \%$ sensitivity and $\sim 90 \%$ specificity (Fig. $3 \mathrm{~b}$ ). Together, these results indicate that CEA-associated glycans represent a powerful tool to distinguish CRC tumor tissues from tumor-adjacent normal tissues with sufficient sensitivity as well as specificity.

\section{Analysis of changes in glycosylation of CEA in colorectal carcinoma with four stages}

In order to investigate the relationship between CEA concentration and CRC stages, we analyzed CEA concentration in serum and tissues with four stages of CRC using a One-way ANOVA test. As shown in Additional file 3, no significant differences in CEA concentration were observed between our biological samples, either serum, CRC tumor tissues or CRC tumor-adjacent normal tissues. Importantly, CEA concentration between samples from different CRC stages showed negligible variation.

To evaluate whether changes in glycan patterns allow for unambiguous identification of the four stages of CRC, we performed statistical analysis of the fold changes of lectins binding to CEA between CRC tumor tissues and paired tumor-adjacent normal tissues using One-way ANOVA test. The fold changes were calculated using the signal-to-noise ratios of tumor tissue samples divided by the signal-to-noise ratios of paired tumoradjacent normal tissue samples, indicated as the value of T/A. As shown in Fig. 4, 11 lectins bound to CEA with significant differences, indicating significant changes in CEA glycan levels of $\mathrm{N}$-acetylgalactosamine, mannose, galactose, $\mathrm{N}$-acetylglucosamine at different CRC stages. Interestingly, the levels of these glycosylation modifications on CEA showed a downward trend following an increase at Stage II. Together, these results indicate that CEA-associated $N$-acetylgalactosamine, mannose, galactose, $N$-acetylglucosamine increase at stage II, before falling to the levels observed at the advanced stage of CRC.

\section{Discussion}

CEA is one of the most widely used protein biomarkers for CRC; however, its clinical use is limited due to its low sensitivity and specificity [4]. Recent studies suggested 


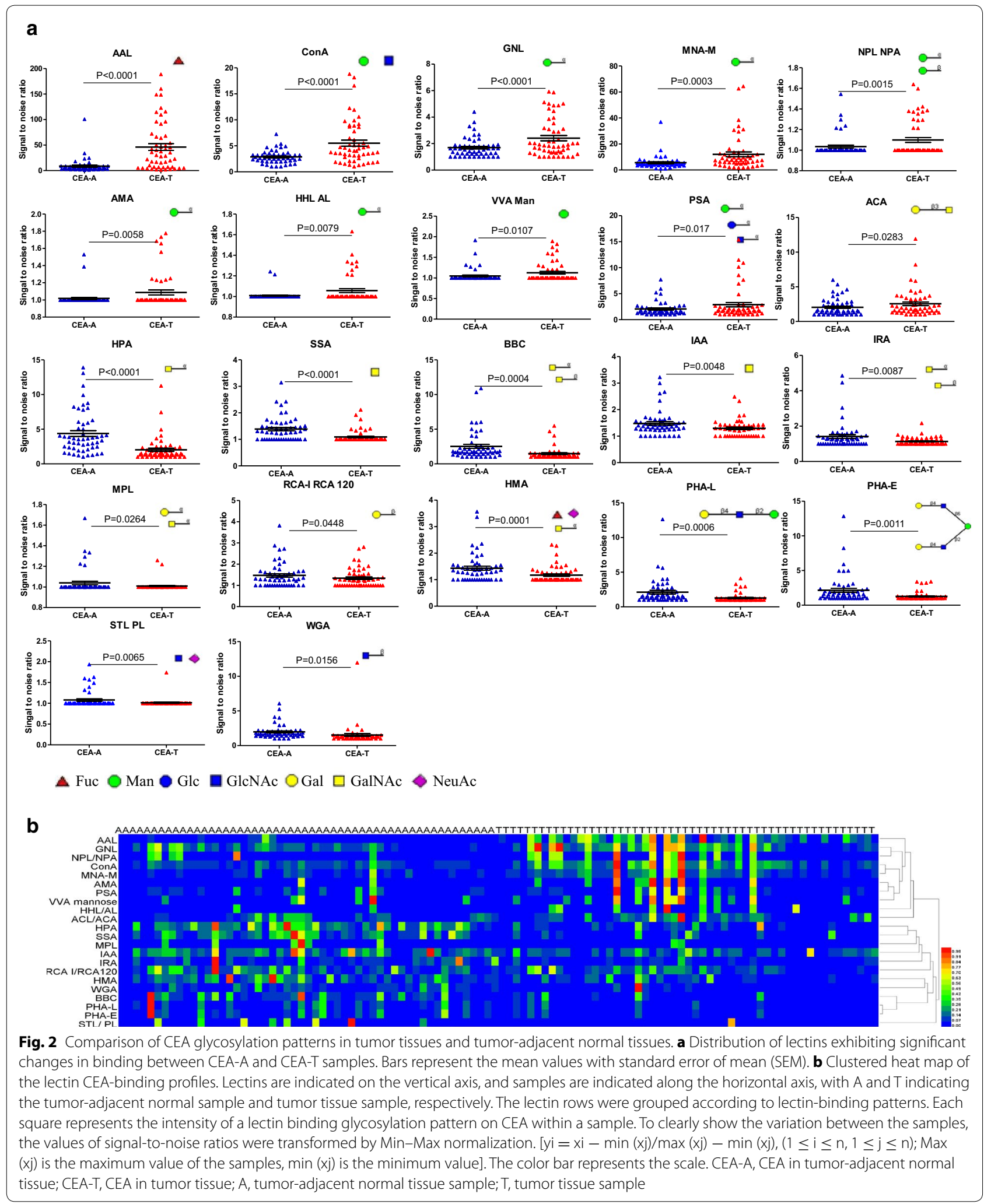




\begin{tabular}{|c|c|c|c|c|c|}
\hline \multirow{2}{*}{ Lectin } & \multirow[t]{2}{*}{ AUC } & \multicolumn{2}{|c|}{$\begin{array}{l}\text { Asympotic } 95 \% \text { confidence } \\
\text { Interval }\end{array}$} & \multirow[t]{2}{*}{ Sensitivity } & \multirow[t]{2}{*}{ Specificity } \\
\hline & & Low Bound & Upper Bound & & \\
\hline HPA & 0.814 & 0.731 & 0.897 & $69.80 \%$ & $84.90 \%$ \\
\hline WGA & 0.795 & 0.706 & 0.884 & $67.90 \%$ & $84.90 \%$ \\
\hline AAL & 0.791 & 0.701 & 0.881 & $67.90 \%$ & $88.70 \%$ \\
\hline PHA-L & 0.761 & 0.667 & 0.856 & $75.50 \%$ & $75.50 \%$ \\
\hline $\mathrm{BBC}$ & 0.760 & 0.665 & 0.854 & $66.00 \%$ & $84.90 \%$ \\
\hline SSA & 0.740 & 0.643 & 0.836 & $64.20 \%$ & $83.00 \%$ \\
\hline MNA-M & 0.727 & 0.625 & 0.828 & $67.90 \%$ & $79.20 \%$ \\
\hline ConA & 0.716 & 0.615 & 0.818 & $67.90 \%$ & $75.50 \%$ \\
\hline PHA-E & 0.710 & 0.610 & 0.811 & $64.20 \%$ & $77.40 \%$ \\
\hline HMA & 0.689 & 0.587 & 0.790 & $62.30 \%$ & $73.60 \%$ \\
\hline IRA & 0.644 & 0.539 & 0.749 & $58.50 \%$ & $67.90 \%$ \\
\hline GNL & 0.642 & 0.536 & 0.748 & $50.90 \%$ & $77.40 \%$ \\
\hline IAA & 0.631 & 0.525 & 0.737 & $36.60 \%$ & $84.90 \%$ \\
\hline ACL ACA & 0.593 & 0.485 & 0.701 & $62.30 \%$ & $56.60 \%$ \\
\hline NPL NPA & 0.582 & 0.473 & 0.691 & $26.40 \%$ & $90.60 \%$ \\
\hline $\begin{array}{l}\text { VVA } \\
\text { mannose }\end{array}$ & 0.576 & 0.467 & 0.685 & $26.40 \%$ & $88.70 \%$ \\
\hline AMA & 0.575 & 0.466 & 0.685 & $18.90 \%$ & $96.20 \%$ \\
\hline $\begin{array}{l}\text { RCAI } \\
\text { RCA120 }\end{array}$ & 0.574 & 0.464 & 0.683 & $56.60 \%$ & $62.30 \%$ \\
\hline HHL AL & 0.568 & 0.459 & 0.678 & $17.00 \%$ & $96.20 \%$ \\
\hline STL PL & 0.565 & 0.455 & 0.674 & $15.10 \%$ & $98.10 \%$ \\
\hline PSA & 0.545 & 0.45 & 0.656 & $60.40 \%$ & $56.60 \%$ \\
\hline MPL & 0.539 & 0.429 & 0.649 & $11.30 \%$ & $96.20 \%$ \\
\hline
\end{tabular}

b

Fig. 3 Receiver operating characteristic (ROC) curves for the discriminating tumor tissue samples from tumor-adjacent normal tissue samples using individual and combined lectins. a The area-under-the-curve (AUC), 95\% confidence interval, sensitivity and specificity of 22 lectins alone. b The $\mathrm{ROC}$ is indicated for combination of 9 lectins

that the detection of glycan variants on a particular protein should yield more effective biomarkers than the measurement of protein concentration [24]. Our findings presented here reveal that glycosylation patterns on CEA differ significantly between CRC tumor tissues and paired tumor-adjacent normal tissues. In addition, we found that the changes of glycosylation levels on CEA correlate with the different stages of CRC.

Here, we used CRC tissues to investigate the changes of glycans on CEA rather than blood and stool samples. Although blood and stool are available and non-invasive to collect, blood is a heterogeneous mixture of proteins derived from different tissues and proteins from stool samples are degraded by the gut micro-biota [8]. Moreover, CEA is commonly detected in a number of tumors of epithelial origin such as lung adenocarcinoma except colorectal carcinoma, in some benign diseases and even in normal adult tissues [26-28], which renders identification of the source of CEA in blood and stool samples difficult. Therefore, a pivotal element of our investigation is the fact that we used tissue samples from CRC patients, ensuring that CEA protein originates in the CRC tumor itself and the detected glycosylations on CEA represent exactly their distribution in CRC patients.

Due to the large quantity of purified glycoprotein required and limited sample throughput, other traditional methods like liquid chromatography (LC), capillary electrophoresis (CE) and MALDI-TOF-MS ${ }^{\mathrm{n}}$ cannot be applied to the analysis of glycan variants on CEA from individual CRC tissues [29]. Therefore, we employed lectin microarray technology to analyze the patterns of CEA glycosylation. To obtain a detailed glycan map of CEA, we applied CRC tissues lysates containing 0.5 $\mu \mathrm{g}$ CEA protein to the microarray. Apart from the small amounts of sample material required, lectin microarray technology possesses the additional advantage of global screening to identify lectins interacting glycoprotein and determining the glycosylation changes with high reproducibility and high sample throughput.

In the present study, we found that fucose (Fuc1-2,3,4) is increased in tumor-associated CEA. Fucosylated glycans can be generally divided into terminal fucosylation (giving rise to specific blood group antigens such as SLe ${ }^{\mathrm{a}}$ and SLe ${ }^{\mathrm{x}}$ ) and core fucosylation(creating a non-extendable modification) [30]. The core fucosylation (CF) of $\mathrm{N}$-glycoproteins plays important roles in regulating protein functions during biological development [31]. Fucosylation levels increase significantly in colon cancer for $\mathrm{N}$-glycans, $\mathrm{O}$-glycans and globo-type glycosphingolipid (GSL) glycans [32]. In agreement with these results, we also observed that fucose(Fuc1-2,3,4) is expressed at higher levels in tumor-ssociated CEA. These elevated levels of fucosylation may be caused by upregulated fucosyltransferase VI, which was reported as a major enzyme modulating the $\mathrm{SLe}^{\mathrm{x}}$ biosynthesis in colorectal cancer [33, 34]. 


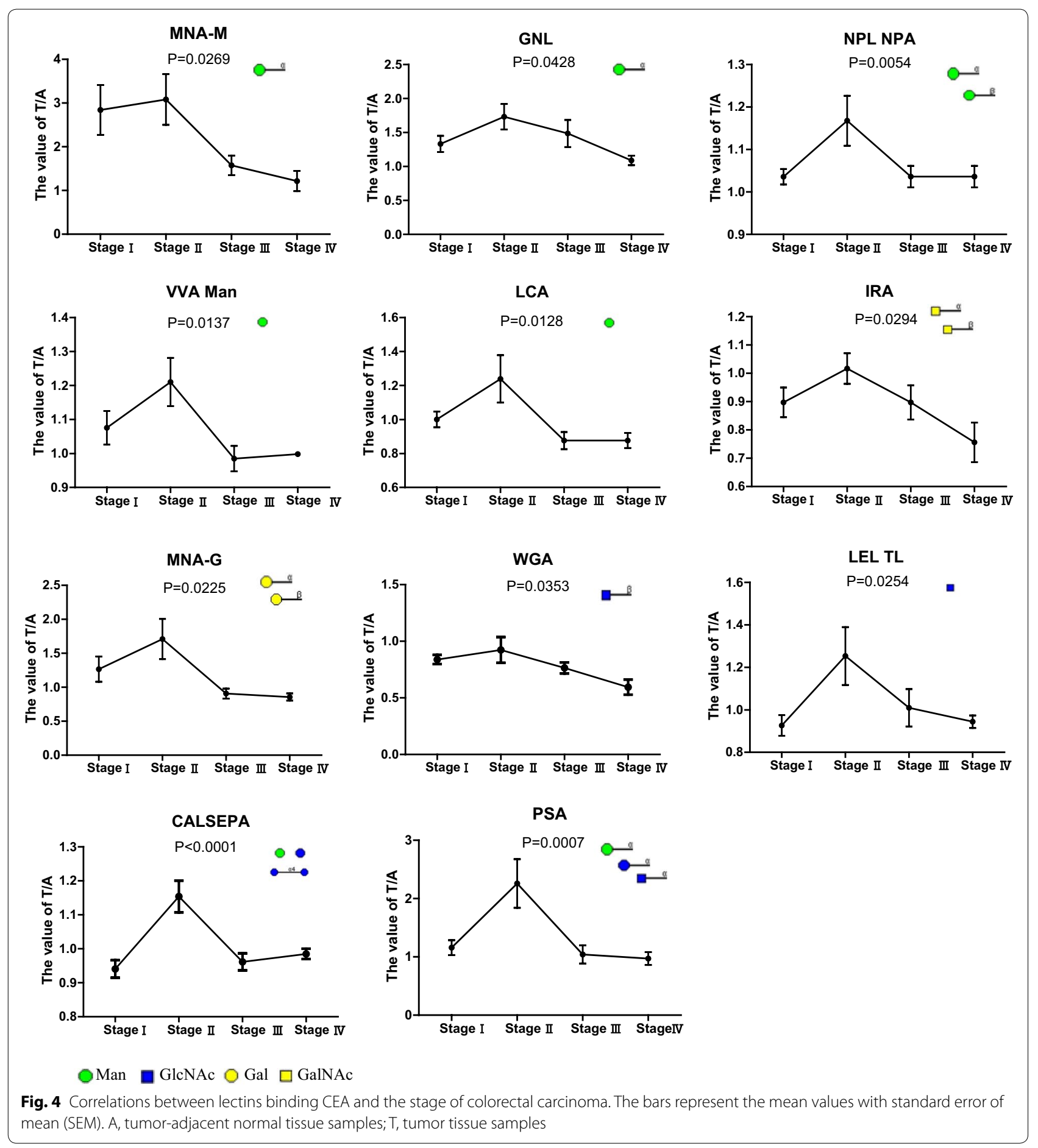

Our results show that mannose (Man) levels are elevated in tumor-associated CEA. Previous studies showed that the cryptic and high-mannose $\mathrm{N}$-glycans emerge in CRC tumor tissues, especially in cell lines [35, 36]. However, while the function of altered mannose in cancer progression remains unclear, it has been suggested earlier that the increase of high-mannose $\mathrm{N}$-glycans in cancer might be the result of precursor accumulation, due to incomplete maturation during $N$-glycan biosynthesis [14, 37].

Furthermore, the Thomsen-Friedenreich antigen (TF-antigen) (Core1, Gal $\beta 1-3$ GalNAc-Ser/Thr) as also 
increased in tumor-associated CEA. TF-antigen has been reported to be associated with metastasis [38]. Saeland et al. [20] found TF-antigen increased on MUC1 from CRC tumor tissue. Recent studies shows that Galectin-3 secreted by tumor cells binds TF-antigen on MUC1 [39, 40]. Due to the interactions between Galectin-3 and TF-antigen, clustered MUC1 on the cell surface exposes adhesion molecules, such as E-Cadherin, avoiding initiation of anoikis (suspension-induced cell death) [39]. It has been shown previously that these exposured adhesion molecules may induce interactions with endothelial cells and promote metastasis [40].

We found that the levels of branched $N$-glycans, bisecting $\mathrm{N}$-glycans and overall $\mathrm{N}$-acetylglucosamine (GlcNAc) are decreased in tumor-associated CEA. Enhanced $\beta 6 \mathrm{GlcNAc}$ side chain branching of $\mathrm{N}$-linked structure (caused by enhanced activity of GnT-V) and counteracting $\beta 4$ GlcNAc (bisecting GlcNAc) (synthesized by GnT$\mathrm{V})$ are the most widely occurring glycosylation changes inducing malignancy [41]. GnT-V promotes metastasis, whereas GnT-III shows the opposite function [13]. Saeland et al. [20] found that branched $N$-glycans were prominently present, however, a change in bisecting $\mathrm{N}$-glycans were not observed on tumor-associated CEA in colorectal cancer tissues. Our results are in contrast with previous studies, which may be caused by sources of material at different disease stage [14]. Therefore, further experiments are required to validate the role of branched $\mathrm{N}$-glycans and bisecting $\mathrm{N}$-glycans in CRC.

In addition, our analysis showed that overall $\mathrm{N}$-acetylgalactosamin (GalNAc) was increased in tumor-associated CEA. GalNAc-type $O$-glycans are found in most transmembrane and secreted glycoproteins. The disaccharide Thomsen-Friedenreich antigen ( $\mathrm{T}$ antigen, also known as core 1) and the mono-saccharide GalNAc (also known as Tn) and their sialylated forms (ST and STn (Neu5Aco2-6GalNAc $\alpha-O-R$ ), respectively) result from the incomplete synthesis of $\mathrm{O}$-glycans. Aberrant glycosylation also occurs in glycoproteins that display abnormal expression of shortened or truncated glycans during malignancy [12].

We also found that galactose (Gal) levels are lower in tumor-associated CEA. Galactosylation is involved in the regulation of immune response by modifying immunoglobulin G (IgG) properties [42]. Low levels of galactose on IgG are associated with a higher proinflammatory activity. The presence of IgG lacking galactose in early synovitis is of prognostic value for the future development of erosive rheumatoid arthritis (RA) [43]. Ruhaak et al. [44] found that galactosylation levels are reduced in the tissue samples of lung adenocarcinoma patients. Down-regulated galactose on CEA may be correlated with CRC immune response.
Importantly, our ROC analysis indicates that $N$-acetylgalactosamin, $N$-acetylglucosamine, fucose, mannose, branched $N$-glycans and bisecting $N$-glycans represent those CEA-associated glycans with the most significant changes. Thus, our analysis provides strong evidence that for clinical purposes, a combination of lectins recognizing these glycans can greatly improve the power of discrimination between CRC tumor tissue from healthy adjacent tissue, making these glycans ideal panel biomarkers for CRC diagnosis.

In our study, we observed that the levels of $\mathrm{N}$-acetylgalactosamine, mannose, galactose, $N$-acetylglucosamine on CEA first increased at Stage II, before falling to their original values or below. This indicates that CEA glycans change dynamically with CRC development, raising the possibility that these altered glycans play transient roles in the progression of tumor. Previous studies proposed that changes in glycan patterns represent a hallmark of cancer progression [14, 45]. However, the precise molecular mechanisms for inducing such changes in CEA glycosylation levels throughout the different stages of CRC remain unclear. A number of reports found that cancer-associated changes in glycan patterns are a result of incomplete synthesis and neo-synthesis processes. More specifically, earlier studies found that incomplete synthesis occurs more often in the early stages of cancer, whereas neo-synthesis is more commonly observed in the advanced stages of cancer [12, 46]. Based on these findings, we hypothesize that levels of immature $\mathrm{N}$-glycan (high-mannose type) and truncated $\mathrm{O}$-glycan ( $N$-acetylgalactosamine) are likely to increase in the early stages of CRC, and then decrease in the later stages of tumor development. This provides an explanation for the changes of the levels of glycosylations on CEA along with the stages of CRC. The differential expression levels of glycotransferase during cancer progression may be another reason for the changes of the levels of glycosylations on CEA with the stages of CRC. Munkley et al. $[47,48]$ reported that the expression of ST6GalNAc1 (the sialyltransferase $\alpha$-GalNAc $\alpha-2,6$-sialyltransferase, an enzyme that catalyses the transfer of a sialic acid molecule in an $\alpha-2-6$ linkage onto the Tn antigen (resulting in GalNAc1-O-serine/threonine)) was increased in primary prostate tumours and decreased in metastatic tissue relative to non-malignant prostate tissue. Further studies will be required to better understand the glycotransferase levels relevant to CEA glycan patterns.

\section{Conclusions}

Our findings provide evidence that analysis of glycan patterns present a reliable and powerful tool for the diagnosis and staging of CRC; in addition, combinatorial analysis of specific glycan profiles possibly allows for 
identification of specific cancer stages. Together, temporal changes in glycan expression on marker proteins such as CEA should allow for the early detection of colorectal carcinoma, as well as lead to a better understanding of the role of CEA in the pathogenesis and progression of colorectal carcinoma.

\section{Additional files}

Additional file 1: Table S1. Lectin used in this study.

Additional file 2. 31 lectins bind different concentrations of CEA.

Additional file 3. The correlation between other diseases and glycans on CEA.

Additional file 4: Table S2. The concentration of CEA of CRC patients; Figure S1. The relationship between CEA concentration and CRC stages.

\begin{abstract}
Abbreviations
CEA: carcinoembryonic antigen; CRC: colorectal cancer; CEA-T: CEA from tumor tissues; CEA-A: CEA from tumor-adjacent normal tissues; TF-antigen: Thomsen-Friedenreich antigen; HCC: hepatocellular carcinoma; UICC: International Union Against Cancer; AJCC: American Joint Committee on Cancer; ELISA: enzyme-linked immunoadsorbent assay; CV: coefficient of variation; SEM: standard error of mean; ANOVA: one-way analysis of variance; A: tumor-adjacent normal tissue samples; T: tumor tissue samples; SD: standard deviation; M: male; F: female; $\mathrm{C}_{(\mathrm{CEA})}$ : the concentration of CEA; ROC: receiver operating characteristic; AUC: area under the curve; LC: liquid chromatography; CE: capillary electrophoresis; MALDI-TOF-MS ${ }^{\text {: }}$ : matrix-assisted laser desorption/ionization-time of flight-mass spectrometry ${ }^{\text {n }}$ : CF: core fucosylation; Fuc: fucose; Man: mannose; Glc: glucose; GlcNAc: N-acetylglucosamine; GalNAc: N-acetylgalactosamin; Gal: galactose; NeuAc: N-acetylneuraminic acid; IgG: immunoglobulin G; RA: rheumatoid arthritis.
\end{abstract}

\section{Authors' contributions}

QZ carried out study design, the lectin microarrays, data analysis and participated in drafting the manuscript. TZ contributed to the isolation and purification of colorectal carcinoma tissue, and provided extensive discussion and clinical information. ZD contributed to study design, data analysis and manuscript writing. QL, YL and SY performed protein extraction, ELISA experiments and quantification of CEA. DJ collected samples from patients, and provided clinical information. YL conceived the study, contributed to its design and coordination, participated in drafting the manuscript and critical review. All authors read and approved the final manuscript.

\section{Author details}

${ }^{1}$ Laboratory of Interdisciplinary Research, Institute of Biophysics, Chinese Academy of Sciences, 15 Datun Road, Chaoyang District, Beijing 100101, China. ${ }^{2}$ University of Chinese Academy of Sciences, Beijing 100049, China. ${ }^{3}$ Key Laboratory of Carcinogenesis and Translational Research (Ministry of Education), Department of Colorectal Surgery, Peking University Cancer Hospital and Institute, Beijing 100142, China. ${ }^{4}$ GuangDong Bio-healtech Advanced Co., Ltd., Foshan 528000, China.

\section{Acknowledgements}

We thank Prof. Shengce Tao for providing technical supports for lectin microarray experiments and Torsten Juelich (Peking University) for linguistic assistance during the preparation of this manuscript. We also thanks Prof. Jinling Zhang and Prof. Ming Li for providing guidance for experiment designing and sample collection.

\section{Competing interests}

The authors declare that they have no competing interests that could be perceived as prejudicing the impartiality of the research reported.
Availability of data and materials

All data are fully available without restriction.

\section{Consent for publication}

Not applicable.

\section{Ethics approval and consent to participate}

Samples and clinical information were reviewed and approved by the Institutional Review Board of Institute of Biophysics, Chinese Academy of Sciences. All patients signed informed consent.

\section{Funding}

This work was supported by the National Natural Science Foundation of China [Grant Number 31270909] and [Grant Number 31700702]; the Major State Basic Research Development Program of China (973 Program) [Grant Number 2013CB910100]; Grant from China Shenzhen City Science and Techology Bureau [Grant Number JCYJ20140416122812021].

\section{Publisher's Note}

Springer Nature remains neutral with regard to jurisdictional claims in published maps and institutional affiliations.

Received: 12 June 2017 Accepted: 23 January 2018

Published online: 02 March 2018

\section{References}

1. Stewart BW, Wild C. World cancer report 2014, Chapter 1.1. Geneva: World Health Organization; 2015.

2. Siegel R, Ms DNM, Dvm AJ. Cancer statistics, 2013. CA Cancer J Clin. 2013;64(1):9-29.

3. Søreide K, et al. Evolving molecular classification by genomic and proteomic biomarkers in colorectal cancer: potential implications for the surgical oncologist. Surg Oncol. 2009;18(1):31-50.

4. Duffy MJ. Carcinoembryonic antigen as a marker for colorectal cancer: is it clinically useful? Clin Chem. 2001:47(4):624-30.

5. Dalerba P, et al. CDX2 as a Prognostic Biomarker in Stage II and Stage III Colon Cancer. N Engl J Med. 2016:374(3):211-22.

6. de Wijkerslooth TR, Bossuyt PM, Dekker E. Strategies in screening for colon carcinoma. Neth J Med. 2011;69(3):112-9.

7. Walther A, et al. Genetic prognostic and predictive markers in colorectal cancer. Nat Rev Cancer. 2009;9(7):489-99.

8. Alvarez-Chaver P, et al. Proteomics for discovery of candidate colorectal cancer biomarkers. World J Gastroenterol. 2014;20(14):3804-24.

9. Arnold JN, et al. The impact of glycosylation on the biological function and structure of human immunoglobulins. Immunology. 2007;25(25):21-50.

10. Roth J. Protein N-glycosylation along the secretory pathway: relationship to organelle topography and function, protein quality control, and cell interactions. Chemlnform. 2002;102(20):285-303.

11. Lowe JB. Glycosylation in the control of selectin counter-receptor structure and function. Immunol Rev. 2002;186(1):19-36.

12. Pinho SS, Reis CA. Glycosylation in cancer: mechanisms and clinical implications. Nat Rev Cancer. 2015;15(9):540-55.

13. Hakomori S. Glycosylation defining cancer malignancy: new wine in an old bottle. Proc Natl Acad Sci USA. 2002;99(16):10231-3.

14. Holst $S$, Wuhrer M, Rombouts Y. Glycosylation characteristics of colorectal cancer. Adv Cancer Res. 2015;126:203-56.

15. Li C, et al. Pancreatic cancer serum detection using a lectin/glyco-antibody array method. J Proteome Res. 2009;8(2):483-92.

16. Zhao J, et al. Glycoprotein microarrays with multi-lectin detection: unique lectin binding patterns as a tool for classifying normal, chronic pancreatitis and pancreatic cancer sera. J Proteome Res. 2007;6(5):1864-74.

17. Sato $Y$, et al. Early recognition of hepatocellular carcinoma based on altered profiles of alpha-fetoprotein. N Engl J Med. 1993;328(25):1802-6.

18. Thomas $P$, et al. The structure, metabolism and function of the carcinoembryonic antigen gene family. Biochem Biophys Acta. 1032;1990(1032):177-89. 
19. Huang $C$, et al. Glycomic profiling of carcinoembryonic antigen isolated from human tumor tissue. Clin Proteomics. 2015;12(1):17.

20. Saeland E, et al. Differential glycosylation of MUC1 and CEACAM5 between normal mucosa and tumour tissue of colon cancer patients. Int J Cancer. 2012;131(1):117-28.

21. Van DW, Havenaar EC, Ec BVDL. Alpha 1-acid glycoprotein (orosomucoid): pathophysiological changes in glycosylation in relation to its function. Glycoconj J. 1995;12(3):227.

22. Haab BB, et al. Glycosylation variants of mucins and CEACAMs as candidate biomarkers for the diagnosis of pancreatic cystic neoplasms. Ann Surg. 2010;251(5):937-45

23. Zhou SM, et al. Lectin RCA-I specifically binds to metastasis-associated cell surface glycans in triple-negative breast cancer. Breast Cancer Res. 2015;17:36.

24. Chen $\mathrm{S}$, et al. Multiplexed analysis of glycan variation on native proteins captured by antibody microarrays. Nat Methods. 2007;4(5):437-44.

25. Li Y, et al. Detection and verification of glycosylation patterns of glycoproteins from clinical specimens using lectin microarrays and lectin-based immunosorbent assays. Anal Chem. 2011;83(22):8509-16.

26. Kim J, et al. Expression of carcinoembryonic antigen and related genes in lung and gastrointestinal cancers. Int J Cancer. 1992;52(5):718-25.

27. Thompson J, et al. A polymerase-chain-reaction assay for the specific identification of transcripts encoded by individual carcinoembryonic antigen (CEA)-gene-family members. Int J Cancer. 1993;55(2):311-9.

28. Boucher D, et al. Studies on the control of gene expression of the carcinoembryonic antigen family in human tissue. Can Res. 1989;49(4):847-52.

29. Nwosu CC, et al. Simultaneous and extensive site-specific N- and O-glycosylation analysis in protein mixtures. J Proteome Res. 2011;10(5):2612-24.

30. Carvalho AS, et al. Differential expression of a-2,3-sialyltransferases and $a-1,3 / 4-$ fucosyltransferases regulates the levels of sialyl Lewis a and sialyl Lewis $\mathrm{x}$ in gastrointestinal carcinoma cells. Int J Biochem Cell Biol. 2009:42(1):80-9.

31. $\mathrm{Ma}$ C, et al. A precise approach in large scale core-fucosylated glycoprotein identification with low- and high-normalized collision energy. J Proteomics. 2015;114:61-70

32. Holst $\mathrm{S}$, et al. Investigations on aberrant glycosylation of glycosphingolipids in colorectal cancer tissues using liquid chromatography and matrixassisted laser desorption time-of-flight mass spectrometry (MALDI-TOFMS). Mol Cell Proteomics MCP. 2013;12(11):3081-93.

33. Trinchera $\mathrm{M}$, et al. The biosynthesis of the selectin-ligand sialyl Lewis $\mathrm{x}$ in colorectal cancer tissues is regulated by fucosyltransferase $\mathrm{VI}$ and can be inhibited by an RNA interference-based approach. Int J Biochem Cell Biol. 2011;43(1):130-9.
34. Miyoshi E, Moriwaki K, Nakagawa T. Biological function of fucosylation in cancer biology. J Biochem. 2008;143(6):725-9.

35. Balog Cl, et al. $\mathrm{N}$-glycosylation of colorectal cancer tissues: a liquid chromatography and mass spectrometry-based investigation. Mol Cell Proteomics. 2012;11(9):571-85.

36. Sethi MK, et al. Comparative $\mathrm{N}$-glycan profiling of colorectal cancer cell lines reveals unique bisecting GlcNAc and a-2,3-linked sialic acid determinants are associated with membrane proteins of the more metastatic/ aggressive cell lines. J Proteome Res. 2014;13(1):277-88.

37. Zhao YY, et al. Functional roles of $\mathrm{N}$-glycans in cell signaling and cell adhesion in cancer. Cancer Sci. 2008;99(7):1304-10.

38. Iwai T, et al. Core 3 synthase is down-regulated in colon carcinoma and profoundly suppresses the metastatic potential of carcinoma cells. Proc Natl Acad Sci. 2005;102(12):4572-7.

39. Zhao Q, et al. Interaction between circulating galectin-3 and cancer-associated MUC1 enhances tumour cell homotypic aggregation and prevents anoikis. Mol Cancer. 2010;9(1):645-61.

40. Yu LG, et al. Galectin-3 interaction with Thomsen-Friedenreich disaccharide on cancer-associated MUC1 causes increased cancer cell endothelial adhesion. J Biol Chem. 2007;282(1):773-81.

41. Pierce $M$, et al. Regulation of $\mathrm{N}$-acetylglucosaminyltransferase $V$ and Asnlinked oligosaccharide beta $(1,6)$ branching by a growth factor signaling pathway and effects on cell adhesion and metastatic potential. Glycoconj J. 1997;14(5):623-30.

42. Huhn C, et al. IgG glycosylation analysis. Proteomics. 2009;9(4):882-913.

43. Young A, et al. Agalactosyl IgG: an aid to differential diagnosis in early synovitis. Arthritis Rheumatol. 1991;34(11):1425-9.

44. Ruhaak LR, et al. Differential $N$-glycosylation patterns in lung adenocarcinoma tissue. J Proteome Res. 2015;14(11):4538-49.

45. Dennis JW, Granovsky M, Warren CE. Glycoprotein glycosylation and cancer progression. Biochem Biophys Acta. 1999;1473(1):21-34.

46. Hakomori SI, Kannagi R. Glycosphingolipids as tumor-associated and differentiation markers. JNCI J Natl Cancer Inst. 1983:71(2):231-51.

47. Munkley J, et al. The androgen receptor controls expression of the cancer-associated sTn antigen and cell adhesion through induction of ST6GalNAc1 in prostate cancer. Oncotarget. 2015;6(33):281-99.

48. Munkley J, Mills IG, Elliott DJ. The role of glycans in the development and progression of prostate cancer. Nat Rev Urol. 2016;13(6):324-33.

\section{Submit your next manuscript to BioMed Central and we will help you at every step:}

- We accept pre-submission inquiries

- Our selector tool helps you to find the most relevant journal

- We provide round the clock customer support

- Convenient online submission

- Thorough peer review

- Inclusion in PubMed and all major indexing services

- Maximum visibility for your research

Submit your manuscript at www.biomedcentral.com/submit
BioMed Central 